\title{
Integrable Boundaries, Conformal Boundary Conditions and $A-D-E$ Fusion Rules
}

\author{
Roger E. Behrend and Paul A. Pearce \\ Department of Mathematics and Statistics \\ University of Melbourne, Parkville, Victoria 3052, Australia \\ Jean-Bernard Zuber \\ Service de Physique Théorique \\ CEA-Saclay, 91191 Gif-sur-Yvette, Cedex, France
}

(June 18, 2018)

The $s l(2)$ minimal theories are labelled by a Lie algebra pair $(A, G)$ where $G$ is of $A-D$ - $E$ type. For these theories on a cylinder we conjecture a complete set of conformal boundary conditions labelled by the nodes of the tensor product graph $A \otimes G$. The cylinder partition functions are given by fusion rules arising from the graph fusion algebra of $A \otimes G$. We further conjecture that, for each conformal boundary condition, an integrable boundary condition exists as a solution of the boundary Yang-Baxter equation for the associated lattice model. The theory is illustrated using the $\left(A_{4}, D_{4}\right)$ or 3 -state Potts model.

PACS numbers: 11.25.Hf, 05.50.+q, 75.10.Hk

\section{INTRODUCTION}

The study of conformal boundary conditions [1] continues to be an active area of research with applications in statistical mechanics and string theory. The problem of a general classification of conformal boundary conditions has seen a revival of interest recently. For theories with a diagonal torus partition function it is known that there is a conformal boundary condition associated to each operator appearing in the theory. Moreover, the fusion rules of these boundary operators are just given by the bulk fusion algebra and thus by the Verlinde formula [2]. In contrast, for non-diagonal theories, the fusion rules are not known in general and it is not even known what constitutes a complete set of conformal boundary conditions. Indeed, these questions have only been resolved 3,4 4 very recently for the simplest non-diagonal theory, namely, the critical 3-state Potts model. In this letter we conjecture a complete set of conformal boundary conditions, fusion rules and cylinder partition functions for the $s l(2)$ minimal models.

The $s l(2)$ minimal models in the bulk are classified [5] by a pair of simply laced Dynkin diagrams $(A, G)$ of type

$$
(A, G)=\left\{\begin{array}{l}
\left(A_{h-1}, A_{g-1}\right) \\
\left(A_{h-1}, D_{(g+2) / 2}\right), \quad g \text { even } \\
\left(A_{h-1}, E_{6}\right), \quad g=12 \\
\left(A_{h-1}, E_{7}\right), \quad g=18 \\
\left(A_{h-1}, E_{8}\right), \quad g=30
\end{array}\right.
$$

Here $h$ and $g$ are the coprime Coxeter numbers of $A$ and $G$ and the central charges are

$$
c=1-\frac{6(h-g)^{2}}{h g} \text {. }
$$

We conjecture that for these theories a complete set of conformal boundary conditions $i$ and the corresponding boundary operators $\hat{\varphi}_{i}$ are labelled by $i \in(A, G)$

$$
\hat{\varphi}_{i}: \quad i=(r, a) \in(A, G)
$$

where $r, a$ are nodes on the Dynkin diagram of $A$ and $G$ respectively. We will use $G$ to denote the Dynkin diagram and the adjacency matrix of this graph. We use $r, r_{1}, r_{2}$ to denote nodes of $A_{h-1} ; s, s_{1}, s_{2}$ for the nodes of $A_{g-1}$; $a, a_{1}, a_{2}, b$ for the nodes of $G$ and $i, j$ to label nodes in the pair $(A, G)$.

We now introduce fused adjacency matrices (intertwiners) and graph fusion matrices. The fused adjacency matrices $V_{s}$ with $s=1, \ldots, g-1$ are defined recursively by the $\operatorname{sl}(2)$ fusion algebra

$$
V_{s}=V_{2} V_{s-1}-V_{s-2}
$$

subject to the initial conditions $V_{1}=I$ and $V_{2}=G$. The matrices $V_{s}$ are symmetric and mutually commuting with entries given by a Verlinde-type formula

$$
V_{s a}{ }^{b}=\left(V_{s}\right)_{a}{ }^{b}=\sum_{m \in \operatorname{Exp}(G)} \frac{\tilde{S}_{s m}}{\tilde{S}_{1 m}} \Psi_{a m} \Psi_{b m}^{*}
$$

where the columns of the unitary matrices $\tilde{S}$ and $\Psi$ are the eigenvectors of the adjacency matrices $A_{g-1}$ and $G$ respectively and the sum is over the Coxeter exponents of $G$ with multiplicities. We assume the graph $G$ has a distinguished endpoint node labelled $a=1$ such that $\Psi_{1 m}>0$ for all $m$. This is at least the case for $A-D-E$ graphs. In this notation we define the fundamental intertwiner as $\hat{V}_{s}{ }^{a}=V_{s 1}{ }^{a}$. 
The graph fusion matrices $\hat{N}_{a}$ with $a \in G$ were introduced by Pasquier [6]. These are defined by the Verlindetype formula [7]

$\hat{N}_{a b}{ }^{c}=\left(\hat{N}_{a}\right)_{b}{ }^{c}=\sum_{m \in \operatorname{Exp}(G)} \frac{\Psi_{a m} \Psi_{b m} \Psi_{c m}^{*}}{\Psi_{1 m}}, \quad a, b, c \in G$.

These matrices satisfy the matrix recursion relation

$$
G \hat{N}_{a}=\sum_{b \in G} G_{a}{ }^{b} \hat{N}_{b}
$$

and initial conditions $\hat{N}_{1}=I$ and $\hat{N}_{2}=G$ where 2 denotes the unique node adjacent to 1 . The numbers $\hat{N}_{a b}{ }^{c}$ are the structure constants of the graph fusion algebra

$$
\hat{N}_{a} \hat{N}_{b}=\sum_{c \in G} \hat{N}_{a b}^{c} \hat{N}_{c}
$$

All the entries of the fused adjacency matrices $V_{s}$ are nonnegative integers. For a proper choice of the eigenvectors and of the node 1 , the entries of the graph fusion matrices $\hat{N}_{a}$ are also integers, and with the exception of $D_{2 n+1}$ and $E_{7}$, they are nonnegative. A key identity relating the fused adjacency matrices and graph fusion matrices is

$$
V_{s} \hat{N}_{a}=\sum_{b \in G} V_{s a}{ }^{b} \hat{N}_{b} .
$$

\section{FUSION RULES}

Let $i_{1}, i_{2}$ and $i_{3} \in(A, G)$ and consider the tensor product graph $A \otimes G$ with distinguished node $i=1$ given by $i=(r, a)=(1,1)$. Then we conjecture that the fusion rules for the boundary operators (1.3) are

$$
\hat{\varphi}_{i_{1}} \times \hat{\varphi}_{i_{2}}=\sum_{i_{3} \in(A, G)} \mathcal{N}_{i_{1} i_{2}}{ }^{i_{3}} \hat{\varphi}_{i_{3}}
$$

where $\mathcal{N}_{i_{1}}$ are just the graph fusion matrices associated with the tensor product graph $A \otimes G$

$$
\mathcal{N}_{i_{1} i_{2}}{ }^{i_{3}}=\mathcal{N}_{\left(r_{1}, a_{1}\right)\left(r_{2}, a_{2}\right)}{ }^{\left(r_{3}, a_{3}\right)}=N_{r_{1} r_{2}}{ }^{{ }^{r_{3}}} \hat{N}_{a_{1} a_{2}}{ }^{a_{3}}
$$

where $N_{r_{1}}$ are the graph fusion matrices for $A_{h-1}$. Let $\varphi_{r, s}$ be the primary chiral fields with respect to the Virasoro algebra. Then the operators $\hat{\varphi}_{i}=\hat{\varphi}_{r, a}$ are related to $\varphi_{r, s}$ by the intertwining relation

$$
\sum_{b \in G} \hat{\varphi}_{r, b}\left(\hat{V}^{T} \hat{V}\right)_{b}^{a}=\sum_{s \in A_{g-1}} \varphi_{r, s} \hat{V}_{s}^{a}
$$

where $\hat{V}$ is the fundamental adjacency matrix intertwiner defined in sec. I. By equality in (2.3) we mean that the operators on either side satisfy the same algebra under fusion.

We define a conjugation operator $C(a)=a^{*}$ to be the identity except for $D_{4 n}$ graphs where the eigenvectors $\Psi_{a m}$ are complex and conjugation corresponds to the $\mathbb{Z}_{2}$ Dynkin diagram automorphism. It then follows that $\hat{N}_{a^{*} b}{ }^{c}=\hat{N}_{c a}{ }^{b}$. We conjecture that the coefficients of the cylinder partition functions $Z_{i_{1} \mid i_{2}}$ of the $s l(2)$ minimal theories are given by the fusion product $\hat{\varphi}_{i_{1}}^{\dagger} \times \hat{\varphi}_{i_{2}}$, that is

$$
Z_{i_{1} \mid i_{2}}(q)=\sum_{i_{3} \in(A, G)} \mathcal{N}_{i_{1}^{*} i_{2}}^{i_{3}} \hat{\chi}_{i_{3}}(q) .
$$

More explicitly,

$$
\begin{aligned}
& \sum_{\left(r_{3}, a_{3}\right) \in\left(A_{h-1}, G\right)}^{Z_{\left(r_{1}, a_{1}\right) \mid\left(r_{2}, a_{2}\right)}(q)} \mathcal{N}_{\left(r_{1}, a_{1}^{*}\right)\left(r_{2}, a_{2}\right)}{ }^{\left(r_{3}, a_{3}\right)} \hat{\chi}_{r_{3}, a_{3}}(q) \\
= & \sum_{(r, s) \in\left(A_{h-1}, A_{g-1}\right)} \chi_{r, s}(q) N_{r r_{1}}{ }^{r_{2}} V_{s a_{1}}{ }^{a_{2}}
\end{aligned}
$$

where, in terms of Virasoro characters,

$$
\hat{\chi}_{r, a}(q)=\sum_{s \in A_{g-1}} \chi_{r, s}(q) \hat{V}_{s}^{a} .
$$

The equivalence of the two forms (2.4b) and 2.4c of the cylinder partition functions follows from the identity (1.9) with $a=1$. The result (2.4) is not entirely new but generalizes and encompasses several previous results [8, 1.,9]. Note that the matrices $N_{r} \otimes V_{s}$ form a representation of the fusion algebra of the minimal model.

\section{CRITICAL 3-STATE POTTS}

As an example we consider the $\mathcal{M}\left(A_{4}, D_{4}\right)$ or critical 3state Potts model. To avoid redundancy, we consider the folded $\left(T_{2}, D_{4}\right)$ model as shown graphically in Figure 1.

The complete list [3, [] of conformal boundary conditions, conjugate fields $\hat{\varphi}$ and associated characters $\hat{\chi}$ is

$$
\begin{aligned}
& A=(1,1)=(4,1) \quad \hat{\varphi}_{1,1}=I \quad \chi_{0}+\chi_{3} \\
& B=(1,3)=(4,3) \quad \hat{\varphi}_{1,3}=\psi \quad \chi_{2 / 3} \\
& C=(1,4)=(4,4) \quad \hat{\varphi}_{1,4}=\psi^{\dagger} \quad \chi_{2 / 3} \\
& B C=(2,1)=(3,1) \quad \hat{\varphi}_{2,1}=\epsilon \quad \chi_{2 / 5}+\chi_{7 / 5} \\
& A C=(2,3)=(3,3) \quad \hat{\varphi}_{2,3}=\sigma \quad \chi_{1 / 15} \\
& A B=(2,4)=(3,4) \quad \hat{\varphi}_{2,4}=\sigma^{\dagger} \quad \chi_{1 / 15} \\
& F=(1,2)=(4,2) \quad \hat{\varphi}_{1,2}=\eta \quad \chi_{1 / 8}+\chi_{13 / 8} \\
& N=(2,2)=(3,2) \quad \hat{\varphi}_{2,2}=\xi \quad \chi_{1 / 40}+\chi_{21 / 40}
\end{aligned}
$$

The fused adjacency matrices of $G=D_{4}$ are

$$
\begin{aligned}
V_{1}=V_{5} & =\left(\begin{array}{llll}
1 & 0 & 0 & 0 \\
0 & 1 & 0 & 0 \\
0 & 0 & 1 & 0 \\
0 & 0 & 0 & 1
\end{array}\right), \quad V_{2}=V_{4}=\left(\begin{array}{llll}
0 & 1 & 0 & 0 \\
1 & 0 & 1 & 1 \\
0 & 1 & 0 & 0 \\
0 & 1 & 0 & 0
\end{array}\right), \\
& V_{3}= \\
& =\left(\begin{array}{llll}
0 & 0 & 1 & 1 \\
0 & 2 & 0 & 0 \\
1 & 0 & 0 & 1 \\
1 & 0 & 1 & 0
\end{array}\right) .
\end{aligned}
$$




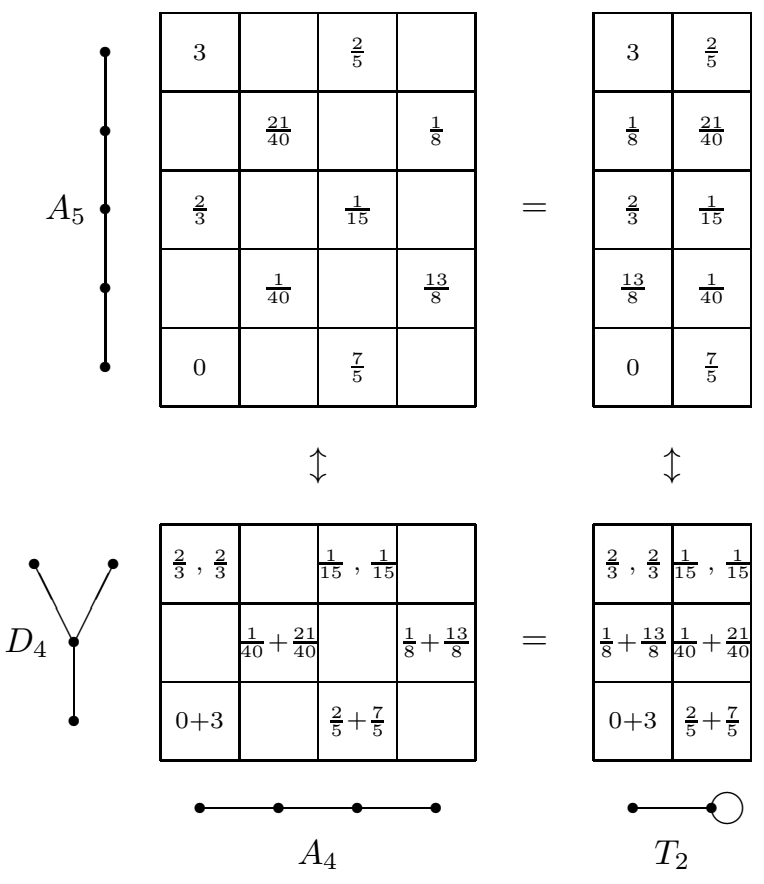

FIG. 1. Folding and orbifold duality relating the tensor product graph $T_{2} \otimes D_{4}$ to $A_{4} \otimes D_{4}$ and $A_{4} \otimes A_{5}$. The conformal weights of the 8 conformal boundary conditions of the 3 -state Potts model appear in the boxes of the $T_{2} \otimes D_{4}$ theory.

The unitary matrix which diagonalizes $D_{4}$ is

$$
\Psi=\frac{1}{\sqrt{3}}\left(\begin{array}{cccc}
\frac{1}{\sqrt{2}} & \frac{1}{\sqrt{2}} & 1 & 1 \\
\sqrt{\frac{3}{2}} & -\sqrt{\frac{3}{2}} & 0 & 0 \\
\frac{1}{\sqrt{2}} & \frac{1}{\sqrt{2}} & \omega & \omega^{2} \\
\frac{1}{\sqrt{2}} & \frac{1}{\sqrt{2}} & \omega^{2} & \omega
\end{array}\right)
$$

where $\omega=\exp (2 \pi i / 3)$ is a primitive cube root of unity. The graph fusion matrices of $D_{4}$ are

$$
\begin{array}{lll}
\hat{N}_{1}=\left(\begin{array}{llll}
1 & 0 & 0 & 0 \\
0 & 1 & 0 & 0 \\
0 & 0 & 1 & 0 \\
0 & 0 & 0 & 1
\end{array}\right), & \hat{N}_{2}=\left(\begin{array}{llll}
0 & 1 & 0 & 0 \\
1 & 0 & 1 & 1 \\
0 & 1 & 0 & 0 \\
0 & 1 & 0 & 0
\end{array}\right), \\
\hat{N}_{3}=\left(\begin{array}{llll}
0 & 0 & 1 & 0 \\
0 & 1 & 0 & 0 \\
0 & 0 & 0 & 1 \\
1 & 0 & 0 & 0
\end{array}\right), & \hat{N}_{4}=\left(\begin{array}{llll}
0 & 0 & 0 & 1 \\
0 & 1 & 0 & 0 \\
1 & 0 & 0 & 0 \\
0 & 0 & 1 & 0
\end{array}\right) .
\end{array}
$$

The graph fusion matrices of $T_{2}$ are

$$
N_{1}=N^{1}=\left(\begin{array}{ll}
1 & 0 \\
0 & 1
\end{array}\right), \quad N_{2}=N^{2}=\left(\begin{array}{ll}
0 & 1 \\
1 & 1
\end{array}\right) .
$$

The intertwiner $\hat{V}$ and conjugation $C$ are

$$
\hat{V}=\left(\begin{array}{cccc}
1 & 0 & 0 & 0 \\
0 & 1 & 0 & 0 \\
0 & 0 & 1 & 1 \\
0 & 1 & 0 & 0 \\
1 & 0 & 0 & 0
\end{array}\right), \quad C=\left(\begin{array}{cccc}
1 & 0 & 0 & 0 \\
0 & 1 & 0 & 0 \\
0 & 0 & 0 & 1 \\
0 & 0 & 1 & 0
\end{array}\right) .
$$

The conjugation operator $C$ acts on the right to raise and lower indices in the fusion matrices $\hat{N}^{a}=\hat{N}_{a} C$.

The complete fusion rules of boundary fields are given as follows:

$$
\begin{aligned}
& \left(\begin{array}{cccccccc}
I & \epsilon & \eta & \xi & \psi & \sigma & \psi^{\dagger} & \sigma^{\dagger} \\
\epsilon & \epsilon^{2} & \epsilon \eta & \epsilon \xi & \epsilon \psi & \epsilon \sigma & \epsilon \psi^{\dagger} & \epsilon \sigma^{\dagger} \\
\eta & \eta \epsilon & \eta^{2} & \eta \xi & \eta \psi & \eta \sigma & \eta \psi^{\dagger} & \eta \sigma^{\dagger} \\
\xi & \xi \epsilon & \xi \eta & \xi^{2} & \xi \psi & \xi \sigma & \xi \psi^{\dagger} & \xi \sigma^{\dagger} \\
\psi & \psi \epsilon & \psi \eta & \psi \xi & \psi^{2} & \psi \sigma & \psi \psi^{\dagger} & \psi \sigma^{\dagger} \\
\sigma & \sigma \epsilon & \sigma \eta & \sigma \xi & \sigma \psi & \sigma^{2} & \sigma \psi^{\dagger} & \sigma \sigma^{\dagger} \\
\psi^{\dagger} & \psi^{\dagger} \epsilon & \psi^{\dagger} \eta & \psi^{\dagger} \xi & \psi^{\dagger} \psi & \psi^{\dagger} \sigma & \psi^{\dagger} & \psi^{\dagger} \sigma^{\dagger} \\
\sigma^{\dagger} & \sigma^{\dagger} \epsilon & \sigma^{\dagger} \eta & \sigma^{\dagger} \xi & \sigma^{\dagger} \psi & \sigma^{\dagger} \sigma & \sigma^{\dagger} \psi^{\dagger} & \sigma^{\dagger^{2}}
\end{array}\right) \\
& =\sum_{r=1}^{2} \sum_{a=1}^{4} N^{r} \otimes \hat{N}^{a} \hat{\varphi}_{r, a} \\
& =\left(\begin{array}{llllllll}
1 & 0 & 0 & 0 & 0 & 0 & 0 & 0 \\
0 & 1 & 0 & 0 & 0 & 0 & 0 & 0 \\
0 & 0 & 1 & 0 & 0 & 0 & 0 & 0 \\
0 & 0 & 0 & 1 & 0 & 0 & 0 & 0 \\
0 & 0 & 0 & 0 & 0 & 0 & 1 & 0 \\
0 & 0 & 0 & 0 & 0 & 0 & 0 & 1 \\
0 & 0 & 0 & 0 & 1 & 0 & 0 & 0 \\
0 & 0 & 0 & 0 & 0 & 1 & 0 & 0
\end{array}\right) I+\left(\begin{array}{llllllll}
0 & 1 & 0 & 0 & 0 & 0 & 0 & 0 \\
1 & 1 & 0 & 0 & 0 & 0 & 0 & 0 \\
0 & 0 & 0 & 1 & 0 & 0 & 0 & 0 \\
0 & 0 & 1 & 1 & 0 & 0 & 0 & 0 \\
0 & 0 & 0 & 0 & 0 & 0 & 0 & 1 \\
0 & 0 & 0 & 0 & 0 & 0 & 1 & 1 \\
0 & 0 & 0 & 0 & 0 & 1 & 0 & 0 \\
0 & 0 & 0 & 0 & 1 & 1 & 0 & 0
\end{array}\right) \epsilon \\
& +\left(\begin{array}{llllllll}
0 & 0 & 1 & 0 & 0 & 0 & 0 & 0 \\
0 & 0 & 0 & 1 & 0 & 0 & 0 & 0 \\
1 & 0 & 0 & 0 & 1 & 0 & 1 & 0 \\
0 & 1 & 0 & 0 & 0 & 1 & 0 & 1 \\
0 & 0 & 1 & 0 & 0 & 0 & 0 & 0 \\
0 & 0 & 0 & 1 & 0 & 0 & 0 & 0 \\
0 & 0 & 1 & 0 & 0 & 0 & 0 & 0 \\
0 & 0 & 0 & 1 & 0 & 0 & 0 & 0
\end{array}\right) \eta+\left(\begin{array}{llllllll}
0 & 0 & 0 & 1 & 0 & 0 & 0 & 0 \\
0 & 0 & 1 & 1 & 0 & 0 & 0 & 0 \\
0 & 1 & 0 & 0 & 0 & 1 & 0 & 1 \\
1 & 1 & 0 & 0 & 1 & 1 & 1 & 1 \\
0 & 0 & 0 & 1 & 0 & 0 & 0 & 0 \\
0 & 0 & 1 & 1 & 0 & 0 & 0 & 0 \\
0 & 0 & 0 & 1 & 0 & 0 & 0 & 0 \\
0 & 0 & 1 & 1 & 0 & 0 & 0 & 0
\end{array}\right) \xi \\
& +\left(\begin{array}{cccccccc}
0 & 0 & 0 & 0 & 1 & 0 & 0 & 0 \\
0 & 0 & 0 & 0 & 0 & 1 & 0 & 0 \\
0 & 0 & 1 & 0 & 0 & 0 & 0 & 0 \\
0 & 0 & 0 & 1 & 0 & 0 & 0 & 0 \\
1 & 0 & 0 & 0 & 0 & 0 & 0 & 0 \\
0 & 1 & 0 & 0 & 0 & 0 & 0 & 0 \\
0 & 0 & 0 & 0 & 0 & 0 & 1 & 0 \\
0 & 0 & 0 & 0 & 0 & 0 & 0 & 1
\end{array}\right) \psi+\left(\begin{array}{llllllll}
0 & 0 & 0 & 0 & 0 & 1 & 0 & 0 \\
0 & 0 & 0 & 0 & 1 & 1 & 0 & 0 \\
0 & 0 & 0 & 1 & 0 & 0 & 0 & 0 \\
0 & 0 & 1 & 1 & 0 & 0 & 0 & 0 \\
0 & 1 & 0 & 0 & 0 & 0 & 0 & 0 \\
1 & 1 & 0 & 0 & 0 & 0 & 0 & 0 \\
0 & 0 & 0 & 0 & 0 & 0 & 0 & 1 \\
0 & 0 & 0 & 0 & 0 & 0 & 1 & 1
\end{array}\right) \sigma \\
& +\left(\begin{array}{cccccccc}
0 & 0 & 0 & 0 & 0 & 0 & 1 & 0 \\
0 & 0 & 0 & 0 & 0 & 0 & 0 & 1 \\
0 & 0 & 1 & 0 & 0 & 0 & 0 & 0 \\
0 & 0 & 0 & 1 & 0 & 0 & 0 & 0 \\
0 & 0 & 0 & 0 & 1 & 0 & 0 & 0 \\
0 & 0 & 0 & 0 & 0 & 1 & 0 & 0 \\
1 & 0 & 0 & 0 & 0 & 0 & 0 & 0 \\
0 & 1 & 0 & 0 & 0 & 0 & 0 & 0
\end{array}\right) \psi^{\dagger}+\left(\begin{array}{cccccccc}
0 & 0 & 0 & 0 & 0 & 0 & 0 & 1 \\
0 & 0 & 0 & 0 & 0 & 0 & 1 & 1 \\
0 & 0 & 0 & 1 & 0 & 0 & 0 & 0 \\
0 & 0 & 1 & 1 & 0 & 0 & 0 & 0 \\
0 & 0 & 0 & 0 & 0 & 1 & 0 & 0 \\
0 & 0 & 0 & 0 & 1 & 1 & 0 & 0 \\
0 & 1 & 0 & 0 & 0 & 0 & 0 & 0 \\
1 & 1 & 0 & 0 & 0 & 0 & 0 & 0
\end{array}\right) \sigma^{\dagger}
\end{aligned}
$$

In total, we find twelve distinct cylinder partition functions [1].3]

$$
\begin{aligned}
Z_{A \mid A}(q)= & \hat{\chi}_{1,1}(q)=\chi_{1,1}(q)+\chi_{1,5}(q) \\
Z_{A \mid B}(q)= & \hat{\chi}_{1,4}(q)=\chi_{1,3}(q) \\
Z_{A \mid A B}(q)= & \hat{\chi}_{2,4}(q)=\chi_{3,3}(q) \\
Z_{A \mid B C}(q)= & \hat{\chi}_{2,1}(q)=\chi_{3,5}(q)+\chi_{3,1}(q) \\
Z_{A \mid F}(q)= & \hat{\chi}_{1,2}(q)=\chi_{4,2}(q)+\chi_{4,4}(q) \\
Z_{A \mid N}(q)= & \hat{\chi}_{2,2}(q)=\chi_{2,2}(q)+\chi_{2,4}(q) \\
= & Z_{A B \mid F}(q) \\
Z_{A B \mid A B}(q)= & \hat{\chi}_{1,1}(q)+\hat{\chi}_{2,1}(q) \\
= & \chi_{1,1}(q)+\chi_{3,5}(q)+\chi_{3,1}(q)+\chi_{1,5}(q) \\
Z_{A B \mid A C}(q)= & \hat{\chi}_{1,4}(q)+\hat{\chi}_{2,3}(q)=\chi_{3,3}(q)+\chi_{1,3}(q) \\
Z_{A B \mid N}(q)= & \hat{\chi}_{2,2}(q)+\hat{\chi}_{1,2}(q) \\
= & \chi_{2,2}(q)+\chi_{2,4}(q)+\chi_{4,2}(q)+\chi_{4,4}(q) \\
Z_{F \mid F}(q)= & \hat{\chi}_{1,1}(q)+\hat{\chi}_{1,3}(q)+\hat{\chi}_{1,4}(q) \\
= & \chi_{1,1}(q)+\chi_{1,5}(q)+2 \chi_{1,3}(q) \\
Z_{F \mid N}(q)= & \hat{\chi}_{2,1}(q)+\hat{\chi}_{2,3}(q)+\hat{\chi}_{2,4}(q) \\
= & \chi_{3,5}(q)+\chi_{3,1}(q)+2 \chi_{3,3}(q) \\
Z_{N \mid N}(q)= & \hat{\chi}_{1,1}(q)+\hat{\chi}_{2,1}(q)+\hat{\chi}_{1,3}(q) \\
& \quad+\hat{\chi}_{1,4}(q)+\hat{\chi}_{2,3}(q)+\hat{\chi}_{2,4}(q)
\end{aligned}
$$




$$
\begin{gathered}
=\chi_{1,1}(q)+\chi_{3,5}(q)+\chi_{3,1}(q)+\chi_{1,5}(q) \\
+2 \chi_{3,3}(q)+2 \chi_{1,3}(q)
\end{gathered}
$$

Here we restrict to Virasoro characters with $r+s$ even. The symmetry

$$
Z_{\left(r_{1}, a_{1}\right) \mid\left(r_{2}, a_{2}\right)}(q)=Z_{\left(r_{2}, a_{2}\right) \mid\left(r_{1}, a_{1}\right)}(q)
$$

follows because the characters do not distinguish between a field $\hat{\varphi}$ and its conjugate $\hat{\varphi}^{\dagger}$.

\section{INTEGRABLE BOUNDARY WEIGHTS}

We conjecture that conformal boundary conditions for $s l(2)$ models can be realized as integrable boundary conditions for the associated lattice models [10]. For the $\left(A_{g-1}, A_{g}\right)$ theories the integrable boundary weights have been obtained [11, as solutions to the boundary YangBaxter equation, by a fusion construction. This method generalizes [11] to the $A-D-E$ models using the appropriate fusion process [12]. The solutions to the boundary Yang-Baxter equation are naturally labelled by a pair $(r, a)$ and are constructed by starting at $a$ and fusing $r-1$ times. For $\left(A_{4}, D_{4}\right)$, the non-zero boundary weights are given explicitly by

$$
\begin{aligned}
& A, B, C=(1, a):{ }_{2}\left\langle\bigsqcup_{a}^{a}=1, \quad a=1,3,4\right. \\
& \left.F=(1,2): 1\rangle_{2}^{2}=3\right\rangle_{2}^{2}=4{ }_{4}^{2}=1 \\
& B C=(2,1): 3\left\langle_{2}^{2}=4\left\langle_{2}^{2}=\rho_{1}(u), 1\left\langle_{2}^{2}=\rho_{1}(-u)\right.\right.\right. \\
& A C=(2,3):{ }_{1}\left\langle\left\langle_{2}^{2}=4\left\langle_{2}^{2}=\rho_{1}(u), 3\left\langle_{2}^{2}=\rho_{1}(-u)\right.\right.\right.\right. \\
& A B=(2,4):{ }_{1}\left\langle\bigsqcup_{2}^{2}=3\left\langle_{2}^{2}=\rho_{1}(u),{ }_{4} \bigsqcup_{2}^{2}=\rho_{1}(-u)\right.\right. \\
& N=(2,2):\left\{\begin{array}{l}
{ }^{2}\left\langle_{a}^{b}=\rho_{2}(u), \quad a \neq b, \quad a, b=1,3,4\right. \\
{ }_{2}^{a}=\rho_{a}(u), \quad a=1,3,4
\end{array}\right.
\end{aligned}
$$

with $u$ the spectral parameter, $\lambda=\pi / 6, \xi$ arbitrary and

$$
\begin{aligned}
& \rho_{1}(u)=\frac{\sin (u-\lambda-\xi) \sin (u-\lambda+\xi)}{\sin ^{2} \lambda}, \quad \rho_{2}(u)=\frac{\sin 2 u}{\sin 2 \lambda} \\
& \rho_{3}(u)=\frac{2 \sin (u-\xi) \sin (u+\xi)+\sin (u-2 \lambda-\xi) \sin (u-2 \lambda+\xi)}{\sin ^{2} 2 \lambda} .
\end{aligned}
$$

The new boundary condition [3] $N$ is found to be antiferromagnetic in nature. The value of $u$ should be set to its isotropic value $u=\lambda / 2$ and $\xi$ chosen appropriately to obtain the conformal boundary conditions.

\section{CONCLUSION}

In conclusion we have proposed a set of conjectures that extend the theory of conformal boundaries in a consistent way. The structure of the partition functions is dictated by a new fusion algebra. We comment that the conjecture (2.4c) is independent of the choice of endpoint node and eigenvectors and is meaningful for $D_{2 n+1}$ and $E_{7}$, even though a proper understanding of the fusion matrices in $(2.4 \mathrm{~b})$ is missing. We expect the extension to higher rank 13 to be straightforward. A much more comprehensive version of this work will be published elsewhere.

[1] J. L. Cardy, Nucl. Phys. B324, 581 (1989).

[2] E. Verlinde, Nucl. Phys. B300, 360 (1988).

[3] I. Affleck, M. Oshikawa and H. Saleur, Boundary Critical Phenomena in the Three-State Potts Model, condmat/9804117

[4] J. Fuchs and C. Schweigert, Completeness of Boundary Conditions for the Critical Three-State Potts Model, hepth/9806121.

[5] A. Cappelli, C. Itzykson and J.-B. Zuber, Comm. Math. Phys. 113, 1 (1987).

[6] V. Pasquier, Modèles Exacts Invariants Conformes, Thèse d'Etat, Orsay, 1988.

[7] P. Di Francesco, and J.-B. Zuber, in Recent Developments in Conformal Field Theories, eds. S. RandjbarDaemi, E. Sezgin and J.-B. Zuber, World Scientific (1990); P. Di Francesco, Int. J. Mod. Phys. A7, 407 (1992).

[8] H. Saleur and M. Bauer, Nucl. Phys. B320, 591 (1989).

[9] V. Pasquier and H. Saleur, Nucl. Phys. B330, 523 (1990).

[10] G. E. Andrews, R. J. Baxter and P. J. Forrester, J. Stat. Phys. 35, 193 (1984); P. J. Forrester and R. J. Baxter, J. Stat. Phys. 38, 435 (1985); V. Pasquier, Nucl. Phys. B285, 162 (1987).

[11] R. E. Behrend, P. A. Pearce and D. L. O'Brien, J. Stat. Phys. 84, 1 (1996); R. E. Behrend and P. A. Pearce, J. Phys. A29, 7827 (1996); R. E. Behrend and P. A. Pearce, Int. J. Mod. Phys. B11, 2833 (1997).

[12] Y.-K. Zhou and P. A. Pearce, Int. J. Mod. Phys. B8, 3531 (1994).

[13] P. Di Francesco, and J.-B. Zuber, Nucl. Phys. B338, 602 (1990); S. Loesch, Y.-K. Zhou and J.-B. Zuber, Int. J. Mod. Phys. A12, 4425 (1997). 